\title{
Pathway-Oriented Concepts in Adjuvant and Neoadjuvant Breast Cancer Therapy
}

\author{
Gunter von Minckwitz ${ }^{a, b, c}$ Caterina Fontanella $a^{a, d}$ \\ ${ }^{a}$ German Breast Group, Neu-Isenburg, \\ bWomen's Hospital, University of Frankfurt, \\ 'Senologic Oncology, Düsseldorf, Germany \\ dDepartment of Oncology, University Hospital of Udine, Italy
}

Endocrine therapy for breast cancer, the first targeted therapy developed in any type of cancer, was used successfully without an established target for more than 50 years before Jensen and Jacobson in 1960 described the estrogen receptor [1]. However, modern targeted therapy first requires detailed molecular characterization and definition of targetable pathways. A clear example was the identification of the human epidermal growth factor receptor 2 (HER2). Historically, the overexpression of the HER2 proteins in breast cancer cells was related to an aggressive natural history, but the introduction of drugs that interrupted the HER2 pathway has completely modified the prognosis of patients with HER2-positive breast cancer and has changed clinical practice [2].

Given the impressive advances obtained by targeting HER2 with trastuzumab, a number of important questions are currently being addressed, including the optimal duration of trastuzumab treatment in the adjuvant setting and the identification of biomarkers for sensitivity or resistance to therapy. As underlined in the review article by Pinto and colleagues [3] in this edition of the journal, in 2012 the HERA trial results failed to demonstrate a survival benefit from 2 years of HER2 receptor blockage compared with 1 year. Moreover, a prolonged exposure to trastuzumab increased the number of secondary cardiac events. Interestingly, the FinHER trial showed a benefit in terms of disease-free survival from 9 weeks of trastuzumab treatment compared with no trastuzumab, which was similar to the magnitude of benefit obtained with 1 year of trastuzumab. However, the PHARE trial so far failed to show non-inferiority of 6 months trastuzumab treatment compared with 12 months, but inferiority was also not observed. The investigators conclude that a duration of 1 year of adjuvant trastuzumab still has to be considered standard of care.

One of the most consistent biomarkers of response to the anti-HER2 treatment is the hormone receptor (HR) status [4]. The molecular mechanism of the HR/HER2 crosstalk is well described by Giuliano and colleagues in a second review article in this issue of BREAST CARE [5]. The authors underline that members of the HER2 pathway can reduce estrogen receptor expression both at mRNA and protein level. Thus, targeting only one of the two pathways may lead to an upregulation of the other one. Conformingly, if we compare the NEOSPHERE and the TBCRC 006 neoadjuvant trials, a 3.5 -fold increase in the rate of pathological complete responses (pCR) was shown by adding endocrine therapy to HER2 blockade in patients with HR-positive/HER2-positive disease.

Other interconnections between different pathways become evident by investigating the mechanisms that drive endocrine resistance. As reported in the third review article of this journal's edition by Hasson and colleagues [6], even if the mechanism of crosstalk between the mTOR and HR pathways are not well described, clinical data support the use of mTOR inhibitors in patients who developed resistance to endocrine treatment. In metastatic breast cancer, the addition of an mTOR inhibitor has shown a gain in survival when combined either with an aromatase inhibitor or with tamoxifen. In the neoadjuvant setting, the mTOR inhibitor everolimus has failed to improve the pCR rate when added to paclitaxel in patients with HER2-negative breast cancer unresponsive to an anthracycline-based chemotherapy [7]. However, consistent with the metastatic setting, everolimus significantly increases the response rate when combined with an aromatase inhibitor. Taken together, the results of these trials strongly suggest that the benefit obtained with mTOR-inhibitors could be dependent on the chosen concomitant therapy, underlining an important crosstalk between the HR and mTOR pathways. However, no specific biomarkers for the use of everolimus have been reported yet.

In conclusion, current treatment options for early breast cancer are moving toward potent targeted therapies that can be tailored to an individual patient's tumor. The list of targets for drug treatment has dramatically increased with a deeper understanding of the molecular pathology of breast cancer. However, the rapidly growing body of new drugs will force us

\section{KARGER}

Fax +497614520714

Information@Karger.com

www.karger.com (c) 2013 S. Karger GmbH, Freiburg

$1661-3791 / 13 / 0084-0246 \$ 38.00 / 0$

Accessible online at:

www.karger.com/brc
Prof. Dr. Gunter von Minckwitz,

GBG Forschungs GmbH

Martin-Behaim-Str. 12

63263 Neu-Isenburg, Germany

gunter.vonminckwitz@germanbreastgroup.de 
to select an adequate biomarker for every targeted therapy, in order to identify the population of patients who benefit most from the treatment and thus to increase the cost-effectiveness ratio.

\section{Disclosure Statement}

GvM received research grants and honoraria from GSK, Novartis, and Roche. CF has no conflict of interests to disclose.

\section{References}

1 Jensen EV, Jacobson HI: Fate of steroid estrogens in target tissues; in: Pincus G, Vollmer EP (eds): Biological Activities of Steroids in Relation to Cancer. New York, Academic Press, 1960, pp 161178.

2 Slamon D, Eiermann W, Robert N, Pienkowski T, Martin M, Press M, Mackey J, Glaspy J, Chan A, Pawlicki M, Pinter T, Valero V, Liu MC, Sauter G von Minckwitz G, Visco F, Bee V, Buyse M, Bendahmane B, Tabah-Fisch I, Lindsay MA Riva A, Crown J; Breast Cancer International Research Group. Adjuvant trastuzumab in HER2positive breast cancer. N Engl J Med 2011;365: 1273-1283.

3 Pinto AC, de Azambuja E, Piccart-Gebhart M How long is enough - optimal timing of anti Her2/ neu therapy in the adjuvant setting in early breast cancer. Breast Care 2013;8: DOI: 10.1159/000354697.

4 von Minckwitz G, Untch M, Blohmer JU, Costa SD, Eidtmann H, Fasching PA, Gerber B, Eiermann W, Hilfrich J, Huober J, Jackisch C, Kaufmann M, Konecny GE, Denkert C, Nekljudova V, Mehta K, Loibl S: Definition and impact of pathologic complete response on prognosis after neoadjuvant chemotherapy in various intrinsic breast cancer subtypes. J Clin Oncol 2012;30:1796-1804.

5 Giuliano M, Trivedi MV, Schiff R: Bidirectional crosstalk between the estrogen receptor and human epidermal growth factor receptor 2 signaling pathways in breast cancer: molecular basis and clinical implications. Breast Care 2013;8: DOI: $10.1159 / 000354253$
6 Hasson SP, Rubinek T, Ryvo L, Wolf I: Endocrine resistance in breast cancer: focus on the phosphatidylinositol 3-kinase/Akt/mammalian target of rapamycin signaling pathway. Breast Care 2013;8: DOI: $10.1159 / 000354757$.

7 Huober J, Fasching PA, Hanusch C, Rezai M, Eidtmann H, Kittel K, Hilfrich J, Schwedler K, Blohmer JU, Tesch H, Gerber B, Höß C, Kümmel S, Mau C, Jackisch C, Khandan F, Costa SD, Krabisch P, Loibl S, Nekljudova V, Untch M, von Minckwitz G Neoadjuvant chemotherapy with paclitaxel and everolimus in breast cancer patients with non-responsive tumours to epirubicin/cyclophosphamide (EC) \pm bevacizumab - results of the randomised GeparQuinto study (GBG 44). Eur J Cancer 2013; 49:2284-2293. 